\title{
Da P\&D à inovação: desafios para o setor elétrico brasileiro
}

\author{
From $R \& D$ to innovation: \\ challenges for the Brazilian electricity sector
}

\author{
Adriana Bin ${ }^{1}$ \\ Maria Isabel Vélez ${ }^{2}$ \\ Ana Flávia Portilho Ferro² \\ Sergio Luiz Monteiro Salles-Filho ${ }^{3}$ \\ Carolina Mattos ${ }^{2}$
}

\begin{abstract}
Resumo: No Brasil, companhias atuantes no setor elétrico (geração, transmissão e distribuição - GTD) são obrigadas a investir pelo menos $1 \%$ de sua receita operacional líquida em pesquisa e desenvolvimento (P\&D) e eficiência energética, obrigação que traz grandes desafios à gestão da pesquisa e da inovação. Inseridas em um setor tipicamente comprador de inovações, essas companhias enfrentam limitações relacionadas à geração de inovações: com frequência, os resultados de projetos de $\mathrm{P} \& \mathrm{D}$ não são implementados, o que aponta para problemas na lógica da política de incentivo para inovação no setor, mas também para ausência de mecanismos robustos de tomada de decisões que apoiem as empresas na geração de benefícios a partir de seus esforços de pesquisa. Este trabalho apresenta uma ferramenta aplicável a empresas do setor elétrico brasileiro com o propósito de caracterizar e propor soluções para superar e/ou prevenir lacunas científicas e tecnológicas, mercadológicas e gerenciais susceptíveis de ocorrência em projetos de $\mathrm{P} \& \mathrm{D}$, no intuito de ampliar a apropriação, adoção e difusão de seus resultados e dos benefícios decorrentes.

Palavras-chave: Inovação; P\&D; Gestão; Lacunas; Transferência de tecnologia.
\end{abstract}

\begin{abstract}
In Brazil, companies operating in the electricity sector must invest at least $1 \%$ of their net operating revenues in $R \& D$ and energy efficiency programs. This obligation challenges their capacity to manage their research and innovation efforts. As these companies belong to a supplier-dominated sector, they face constraints related to the generation of innovations: often the results of $R \& D$ projects are not implemented. This situation reveals not only problems in the sector innovation policy, but also the absence of robust mechanisms to support companies in decisions that can help them generate benefits from research. This work presents a tool developed for the Brazilian electricity companies aiming to characterize and propose solutions to overcome and/or prevent scientific and technology, market and management gaps likely to occur in $R \& D$ projects in order to enlarge the appropriation, adoption and diffusion of their results, and the generation of positive impacts.
\end{abstract}

Keywords: Innovation; R\&D; Management; Gaps; Technology transfer.

\section{Introdução}

Como garantir que os investimentos realizados em atividades de pesquisa e desenvolvimento (P\&D) gerem retornos para uma empresa? Como fazer com que os objetivos destas atividades sejam atingidos e que competências e inovações sejam geradas ao final do processo? Este é um desafio que se coloca para todas as empresas que investem em pesquisa.
No entanto, em alguns setores e em determinados contextos, como é o caso do setor elétrico brasileiro, o desafio torna-se ainda maior, justamente pelo fato de haver um determinante exógeno às empresas que define os montantes que devem ser investidos anualmente em pesquisa e que restringe em parte as possibilidades de apropriação dos benefícios destes investimentos,

\footnotetext{
${ }^{1}$ Faculdade de Ciências Aplicadas - FCA, Universidade Estadual de Campinas - UNICAMP, Campinas, SP, Brasil, e-mail: adriana.bin@fca.unicamp.br

${ }^{2}$ Grupo de Estudos sobre Organização da Pesquisa e da Inovação - GEOPI, Departamento de Política Científica e Tecnológica - DPCT, Instituto de Geociências - IG, Universidade Estadual de Campinas - UNICAMP, Campinas, SP, Brasil, e-mail: velez.misabel@gmail.com; anaflaviaferro@gmail.com; carolina.mattos@gmail.com
}

${ }^{3}$ Departamento de Política Científica e Tecnológica - DPCT, Instituto de Geociências - IG, Universidade Estadual de Campinas UNICAMP, Campinas, SP, Brasil, e-mail: sallesfi@ige.unicamp.br

Recebido em Fev. 25, 2014 - Aceito em Abr. 2, 2015

Suporte financeiro: CPFL Energia. 
criando desencontros estratégicos frequentes entre o que a empresa quer e precisa investir e o que ela legalmente deve investir. Esses desencontros têm, invariavelmente, gerado sobre investimentos, com consequente geração de resultados e tecnologias de prateleira (Pompermayer et al., 2011).

Este trabalho tem como objetivo apresentar uma ferramenta desenvolvida no contexto do setor elétrico nacional para a identificação e superação de brechas (doravante denominadas lacunas) entre os resultados dos investimentos em P\&D e sua apropriação como inovações de produtos, processos e serviços.

Atuando em um setor dominado pelos fornecedores, a maior parte das inovações das empresas de geração, transmissão e distribuição de energia no Brasil vem de aquisições junto a empresas multinacionais de materiais e equipamentos. No entanto, a regulação brasileira para este setor prevê um investimento mínimo e compulsório de $1 \%$ da receita operacional líquida das empresas em programas de $\mathrm{P} \& \mathrm{D}$ e de eficiência energética.

A obrigatoriedade da $\mathrm{P} \& \mathrm{D}$ frente à dinâmica inovativa do setor tem demandado processos de gestão cada vez mais robustos para as empresas deste ramo (Fernandino \& Oliveira, 2010; Boer et al., 2014), especialmente para a internalização dos resultados dos projetos e apropriação de seus benefícios dentro dos limites previstos no marco regulatório. O ponto crítico é justamente garantir que os esforços de $\mathrm{P} \& \mathrm{D}$ mantenham-se alinhados com os objetivos e interesses da empresa em termos de seu posicionamento no mercado.

O que se verifica na prática é que a manutenção deste alinhamento não tem sido trivial (Pompermayer et al., 2011; Amaral 2012). Muitos projetos de P\&D geram resultados que não são incorporados pelas empresas, seja diretamente pela geração de inovações, seja indiretamente pela criação de competências relevantes para a atuação futura das empresas (Defeuilley \& Furtado, 2000). Os motivos para isso são diversos e recaem sobre a natureza da tecnologia que se está desenvolvendo, processos produtivos das empresas, dinâmica do mercado, fluxos de informações entre os parceiros envolvidos, entre outros.

A ferramenta aqui apresentada foi desenvolvida justamente no intuito de apoiar as empresas na identificação e superação destes elementos, em direção a maior efetividade de suas atividades de $\mathrm{P} \& \mathrm{D}$ para a promoção de inovações.

A segunda seção do artigo explica o contexto em que as empresas operam no setor elétrico brasileiro, de forma a explicar os desafios que estão na base de operação da P\&D compulsória e justificar o desenvolvimento da ferramenta. Na terceira seção, são apresentados os principais conceitos teóricos em que a ferramenta se baseia, principalmente os relacionados à gestão de P\&D e transferência de tecnologia. A quarta seção apresenta a metodologia empregada para o desenvolvimento da ferramenta, enquanto a quinta traz o seu detalhamento, indicando seus componentes principais, sua lógica de funcionamento e dois exemplos de aplicação. Finalmente, a sexta e última seção discute a aplicabilidade da ferramenta para as empresas que operam no setor elétrico, assim como desdobramentos para empresas de outros setores e para outros tipos de organizações nos quais o mesmo tipo de problema se verifica.

\section{$2 \mathrm{O}$ setor elétrico brasileiro: contexto para o desenvolvimento de uma ferramenta de gestão da $P \& D$ e inovação}

O mercado brasileiro de energia elétrica opera de forma regulada, contando atualmente com um conjunto de empresas concessionárias com maior ou menor nível de integração nos segmentos de geração, transmissão, distribuição e comercialização (GTDC). A maior parte das companhias que operam nos segmentos de GTD são de grande porte, mas existem também empresas de menor porte e cooperativas de energia elétrica que atendem a microrregiões. Outra característica importante deste setor é a tendência recente de concentração do capital, por meio de fusões e aquisições de empresas, envolvendo grandes grupos controladores (holdings).

No que se refere à dinâmica inovativa do setor, cabe destacar como já indicado na introdução deste trabalho, o papel preponderante da indústria de materiais, sistemas e equipamentos, evidenciando, com base na tipologia tecnológica para o setor de serviços de Miozzo \& Soete (2001) e Castellacci (2008), ambas inspiradas na taxonomia de Pavitt (1984) e Bell \& Pavitt (1993), o perfil predominante de tomador de inovações (supplier dominated). Vale, neste sentido, apontar que, embora haja empresas nacionais, a maior parte dos fornecedores é composta por grandes empresas multinacionais, denotando o caráter de dependência do setor elétrico nacional.

A abordagem apresentada por Malerba \& Orsenigo (1997) relacionando padrões de atividade inovativa e distintos regimes tecnológicos complementa a análise anterior, na medida em que permite deduzir certas limitações das empresas do setor não apenas no que diz respeito à deteç̧ão de oportunidades tecnológicas, mas especialmente em suas possibilidades de garantir boas condições de apropriação dos resultados da inovação (Amaral, 2012). Ou seja, há um elemento intrínseco relacionado com a base de conhecimento e as trajetórias tecnológicas e organizacionais que foram construídas e seguidas ao longo do tempo pelas empresas do setor e que acabam também sendo moldadas pelo marco regulatório (restritivo) no que 
se refere à obtenção de retornos a partir da inovação, como será visto a seguir.

Autores como Margolis \& Kammen (1999) e Jamasb \& Pollitt (2011), estudando investimentos em P\&D em vários países na Europa e nos EUA, argumentam que empresas GTD, por sua estrutura de concorrência baseada em concessões de longo prazo e com elevada imobilização de ativos, economias de escala e perfil tomador de inovações por meio de aquisição de equipamentos e sistemas, têm naturalmente um interesse secundário por esforços internos de P\&D.

No Brasil, a situação não tem sido diferente. Os esforços de P\&D e de inovação nas empresas concessionárias de energia, embora existentes e historicamente caracterizados por ações cooperativas como aquelas que estão na base da criação do Centro de Pesquisas de Energia Elétrica (CEPEL) e por esforços internos de empresas estatais, sempre foram restritos em termos de impactos no desempenho econômico das empresas. A própria natureza desses investimentos é incremental e traz impactos localizados muitas vezes difíceis de serem percebidos. No entanto, e levando em consideração a forte relação entre características dos sistemas nacionais de inovação e os padrões setoriais de inovação demonstrada por Castellacci (2009), no caso brasileiro, há um elemento adicional que torna o interesse pela $\mathrm{P} \& \mathrm{D}$ por parte do setor elétrico ainda mais secundário, que é a política de modicidade tarifária que tende a eliminar os ganhos do inovador.

Desde os anos 1990, o setor elétrico brasileiro passou por mudanças institucionais significativas que culminaram no novo marco regulatório, vigente desde 2004. As reformas carregaram as características de um movimento global de privatização de setores regulados, com importantes impactos nas então operadoras federais e estaduais verticalizadas (Vianna, 2004).

Dentre as principais alterações do Novo Modelo do Setor Elétrico introduzidas em 2004, destacam-se: a substituição do critério utilizado para concessão de novos empreendimentos de geração; a instituição de dois ambientes para celebração de contratos de compra e venda de energia, o Ambiente de Contratação Regulada (ACR), para consumidores cativos, do qual participam agentes de geração e de distribuição de energia elétrica, e o Ambiente de Contratação Livre (ACL), do qual participam geradoras, comercializadoras, importadores, exportadores e consumidores livres; a introdução do princípio da modicidade tarifaria, com base em uma revisão periódica da tarifa a cada quatro ou cinco anos, no intuito de reposicioná-la a partir de uma análise detalhada dos custos, investimentos e receitas das distribuidoras; e, por fim, mudanças na estrutura institucional do setor pela introdução de novos agentes (ANEEL, 2009).
$\mathrm{Na}$ área específica da regulação associada à $\mathrm{P} \& \mathrm{D}$ anterior até o estabelecimento do novo modelo para o setor, o principal ponto foi a introdução da obrigatoriedade de investimento de um percentual mínimo da receita operacional líquida (ROL) das empresas em P\&D e em programas de eficiência energética - PEE (estabelecida inicialmente pela Lei $n^{\circ}$ 9.991/2000; Brasil, 2000). De acordo com a lei, parte dos recursos reservados a $P \& D$ seria gerida internamente pelas empresas, enquanto a outra parte deveria ser destinada ao Fundo Nacional de Desenvolvimento Científico e Tecnológico (FNDCT), sendo os investimentos regulamentados pela Agência Nacional de Energia Elétrica (ANEEL) (Campos, 2007; Cunha et al., 2008). Cabe enfatizar que, ao longo dos últimos 15 anos, desde que a Lei $\mathrm{n}^{\circ} 9.991$ foi estabelecida, ela sofreu algumas modificações que alteraram os percentuais de contribuição para PEE e para a P\&D sob gestão da concessionária, destinada ao FNDCT e também à Empresa de Pesquisa Energética (EPE), do Ministério de Minas e Energia (o que passou a ocorrer a partir de 2003).

Originalmente a proporção era de $0,50 \%$ para P\&D e 0,50\% para PEE para as distribuidoras e de $1 \%$ para $P \& D$ para geradoras e transmissoras. Esta é a mesma proporção praticada atualmente, ainda que tenha havido períodos em que a proporção para P\&D e PEE para as distribuidoras tenha sido de $0,75 \%$ e $0,25 \%$ respectivamente. Uma análise detalhada destas alterações realizada por Boer (2013) indica que as mudanças nos percentuais praticados são a resposta a pressões políticas exercidas por distintos atores em prol ou contra a política de contribuição compulsória, assim como pressões relacionadas ao reconhecimento sobre a importância dos recursos destinados à PEE.

A ideia da regulação da $P \& D$ foi justamente promover a inovação no setor, resultando em sua melhoria operacional e tendo como consequência fundamental a sua contribuição para a modicidade tarifária, elemento importante do novo modelo do setor. No entanto, na prática, isto não tem acontecido. Dado o risco associado ao não cumprimento da legislação (por conta das multas), dada a falta de estímulo para a inovação pela perda dos ganhos do inovador na revisão tarifária e dadas as restrições competitivas do setor e as limitações para se apropriar de resultados da comercialização de tecnologias, a P\&D passou a ser encarada como um "fardo" para muitas empresas (Salles-Filho, 2010).

Amaral (2012) reconhece esta situação ao afirmar que a política de estímulo à $\mathrm{P} \& \mathrm{D}$ no setor elétrico brasileiro não tem sido suficiente para induzir relações mais adequadas entre os agentes envolvidos na geração de inovações, assim como para garantir bons resultados em termos de padrões mais elevados de desenvolvimento tecnológico. Em suma, o que 
o autor afirma é que o setor elétrico não apresenta condições que tornem a concorrência pela inovação um elemento estratégico pelas empresas.

Para cumprir com suas obrigações, escapando do assim chamado "risco regulatório" neste cenário, a solução das empresas do setor tem sido terceirizar em parte seus esforços de $\mathrm{P} \& \mathrm{D}$, contratando grupos de universidades e centros de pesquisa como parceiros, ou mesmo aplicar os recursos destinados à $\mathrm{P} \& \mathrm{D}$ de forma "disfarçada" em outras atividades não caracterizadas como P\&D pelo manual da ANEEL (tais como gestão corporativa, estudos de viabilidade técnico econômica entre outros). Como consequência, as empresas criaram modelos minimalistas de gestão de P\&D e inovação (Boer et al., 2014), sem desenvolver componentes fundamentais relacionados com a capacidade de absorção e com a apropriação dos resultados da $\mathrm{P} \& \mathrm{D}$ e geração de benefícios.

Com base nesta constatação, houve, em 2008, uma nova mudança no marco regulatório, que tentou inserir com mais força o elemento da inovação no esforço do chamado P\&D ANEEL, pela introdução de novas modalidades de projetos mais voltados para a incorporação dos resultados da $\mathrm{P} \& D$ na estrutura produtiva das empresas (projetos do tipo cabeça de série, lote pioneiro e inserção no mercado).

Complementarmente, uma nova mudança na legislação passou a permitir às empresas de transmissão e distribuição se apropriarem de $50 \%$ a $70 \%$ dos benefícios econômicos gerados pelo esforço de $\mathrm{P} \& \mathrm{D}$, desde que se trate de tecnologias a serem comercializadas, mas não utilizadas pelas próprias empresas (para o caso da geração, a apropriação pode ser integral).

Ou seja, criam-se desta forma possibilidades para que receitas provenientes da comercialização de tecnologias não sejam totalmente compartilhadas com os consumidores no processo de revisão tarifária (ANEEL, 2008, 2012) e que sejam revertidas em benefícios diretos para as empresas envolvidas.

Conforme apontado por Salles-Filho (2010), embora importantes, tais medidas não são totalmente efetivas por não remunerarem o esforço da inovação feita para uso interno na empresa (com consequentes ganhos de produtividade e qualidade) que, via de regra, representam a maior parte do resultado do investimento em P\&D. Ao invés disso, estimulam as empresas a comercializar tecnologia, em um mercado de equipamentos para GTD notadamente oligopolizado e no qual a indústria nacional apresenta baixa competitividade.

Independente da efetividade destas medidas, é fato que nos últimos anos as empresas do setor estão empreendendo esforços para tornar mais estratégica a P\&D obrigatória e mais ampliadas suas estruturas de gestão outrora minimalistas, seja no que se refere ao uso interno dos resultados gerados ou à sua comercialização, incluindo, em alguns casos, perspectivas de geração de novos modelos de negócio (Tenório, 2009; Pompermayer et al., 2011).

Para tal, as empresas têm tentado lidar - do ponto de vista gerencial - com o estoque de tecnologias geradas pelo investimento de mais de um bilhão de reais por parte das empresas do setor desde 1998, mas também com a priorização e execução mais adequada dos novos projetos, no intuito de fazer com que seu benefício seja contabilizado pela empresa, pelo setor e pela sociedade. É justamente nesta vertente que o presente trabalho se justifica, uma vez que propõe uma ferramenta que apoia as concessionárias na gestão de seus esforços de P\&D (incluindo, mas não restrito a P\&D ANEEL), com a perspectiva de geração de valor por meio da inovação.

\section{Compreendendo os caminhos da $P \& D$ à inovação: bases para o desenvolvimento de uma ferramenta de gestão da $P \& D$ e inovação}

Desde o início dos anos 1970, uma série de estudos sobre superação de gaps tecnológicos foram desenvolvidos. Na América Latina, e em particular no Brasil, os estudos de Herrera $(1971,1982)$ focaram causas e formas de redução de gaps tecnológicos entre países desenvolvidos e em desenvolvimento, sendo este tema posteriormente disseminado por trabalhos como o de Mansfield (1984) e Perez (1985).

Castellacci (2002) mostra que a perspectiva macro dos estudos de redução de gap tecnológico a partir da década de 1980 esteve voltada originalmente para analisar e explicar as diferenças entre o crescimento econômico dos países a partir das suas capacidades domésticas, especialmente daquelas necessárias para absorver transbordamentos de conhecimento/tecnologia provenientes de outros países. Em outras palavras, tratava-se basicamente da capacidade para levar a cabo, de forma satisfatória, os processos de transferência de tecnologia.

Dentre os pontos mais importantes desta discussão, destaca-se a noção de que as reduções de gaps dependem de esforços substantivos da parte receptora, que deve ter papel ativo na construção de competências capazes não somente de operar, mas de reproduzir e avançar no desenvolvimento, promovendo o que se convencionou chamar de catching-up (Abramovitz, 1986; Perez \& Soete, 1988). No centro da discussão, o ponto fundamental foi o de construir capacidades internas de absorção e, em seguida, dar saltos de desenvolvimento.

Apesar de a abordagem de redução de gap ter sido originalmente aplicada na literatura sobre transferência de tecnologia entre países (o que poderíamos chamar de nível "macro" da abordagem), ela pode ser aplicável 
também ao nível "micro", entre organizações ou mesmo entre distintas unidades de uma mesma organização, o que a torna útil na presente discussão sobre a gestão da $\mathrm{P} \& \mathrm{D}$ em empresas do setor elétrico brasileiro. A ideia de capacidade interna de absorção pode ser aplicada neste contexto para o nível da firma e compreendida como sua habilidade de adquirir conhecimento externo (ou de outras unidades da mesma organização), assim como a habilidade de explorar adequadamente este conhecimento assimilado (Cohen \& Levinthal, 1990).

Sob esta perspectiva ampla que abrange os níveis "macro" e "micro", a transferência de tecnologia pode ser definida como o processo por meio do qual um conjunto de conhecimentos tácitos e codificados, know-how, métodos, procedimentos e/ou dispositivos físicos e equipamentos, todos eles aplicáveis à produção, organização e comercialização de bens e serviços, são transferidos de uma organização ou unidade (provedora) para outra (receptora).

Amesse \& Cohendet (2001) indicam quatro situações nas quais se aplica a ideia de transferência de tecnologia e redução de gaps no nível "micro". Duas delas dizem respeito à transferência de resultados da P\&D gerados internamente à empresa, seja para sua utilização em processos produtivos da própria empresa, seja para sua comercialização no mercado (diretamente ou via licenciamento para terceiros, geração de spin-offs, joint ventures, contratos de terceirização da produção entre outros arranjos). As outras duas situações envolvem a absorção dos resultados da P\&D contratada externamente ou mesmo de tecnologias compradas de terceiros, por meio de adaptações necessárias para o seu efetivo uso em processos da própria empresa ou, no caso da aquisição de $\mathrm{P} \& \mathrm{D}$, para sua comercialização no mercado.

Segundo Amesse \& Cohendet (2001) e outros autores que trabalharam este tema de forma aplicada, tais como Rice et al. (2002), Wigren-Kristoferson et al. (2011) e Youtie et al. (2012), a superação destes gaps passa por um conjunto variado de ações que envolvem o desenvolvimento de estratégias para a vinculação dos esforços de $\mathrm{P} \& \mathrm{D}$ da empresa a suas estratégias corporativas, o envolvimento de unidades organizacionais potencialmente usuárias dos resultados da P\&D no desenvolvimento dos projetos, a gestão adequada da estrutura, dos recursos e das competências de $\mathrm{P} \& \mathrm{D}$, o estabelecimento de relacionamentos e fluxos de conhecimento adequados entre a empresa e seus parceiros, entre outras.

Todavia, se por um lado as soluções para a superação de gaps passam por um gerenciamento adequado dos projetos de $\mathrm{P} \& \mathrm{D}$, respeitando seus distintos objetivos e arranjos, por outro há decisões anteriores igualmente importantes, orientadas à melhor escolha entre desenvolver uma dada tecnologia internamente, desenvolvê-la em parceria ou ainda comprá-la - conhecida na literatura como a decisão make or buy.

De acordo com Teece (2010), a aplicação da abordagem dos custos de transação para o universo da gestão, baseada na ponderação dos riscos associados aos arranjos contratuais, modelos híbridos ou à organização hierárquica interna, é extremamente adequada para definir se um determinado investimento deve ser realizado internamente ou se deve ser terceirizado. Para o problema aqui tratado, trata-se de definir se o melhor arranjo passa por uma P\&D in house, por sua contratação externa ou execução em parceria ou se pela aquisição direta da tecnologia.

Nestes dois pontos, a literatura de gestão da inovação e também de inovação aberta tem bastante a contribuir. Há um conjunto grande de trabalhos que discute e apresenta ferramentas para a gestão de portfólio e projetos de $\mathrm{P} \& \mathrm{D}$, de tecnologia e de inovação em suas diferentes vertentes (Cooper, 2000; Tidd et al., 2005; Phaal et al., 2006; Dodgson et al., 2008), trabalhos que estão principalmente orientados à discussão sobre a seleção e gestão de parceiros e arranjos para a execução da P\&D e para o desenvolvimento de capacidades de absorção de conhecimento (Chesbrough, 2006; Gomes et al., 2011; Lavie et al., 2012) ou ainda direcionados à discussão sobre o licenciamento de tecnologias desenvolvidas internamente à organização (Chesbrough, 2006; Lichtenthaler, 2011).

Contudo, apesar da contribuição desta literatura, não foram encontradas evidências de métodos ou ferramentas que apoiem de forma integrada, para uma dada tecnologia tida como prioritária, a decisão make or buy e a decisão de adoção, apropriação e difusão da tecnologia, compreendida como fundamentais para o problema da gestão da $\mathrm{P} \& \mathrm{D}$ e inovação no setor elétrico brasileiro.

Como visto anteriormente, a $\mathrm{P} \& \mathrm{D}$ não tem ainda ocupado um papel estratégico nas empresas deste setor e o equacionamento desta situação vai além de promover um melhor gerenciamento do relacionamento com os parceiros e garantia de alcance dos resultados previstos. Ele inclui necessariamente uma expansão do modelo centrado na terceirização para um modelo de arranjos mistos em que a terceirização da $P \& D$ é uma das vias possíveis. Neste ponto, o nível de maturidade e outras características da tecnologia de interesse, assim como a disponibilidade de competências para desenvolvê-las (interna ou externamente à empresa) são pontos-chave.

Tal mudança passa também pela ampliação da perspectiva sobre os resultados da $\mathrm{P} \& \mathrm{D}$, hoje orientada à geração de tecnologias, para a perspectiva de adoção e comercialização destas tecnologias. Neste ponto, os aspectos gerenciais internos relacionados com os processos organizacionais nos quais estas tecnologias possam ser aplicadas, além da perspectiva comercial 
relacionada com a existência de mercado e de atores interessados em sua produção e comercialização são fundamentais e devem ser levados em conta, mesmo tendo em vista os limites de apropriação de resultados quando se aborda a P\&D regulada e compulsória. São estes os elementos orientadores da ferramenta desenvolvida no âmbito do presente trabalho, como se verá a seguir.

\section{Metodologia: o desenvolvimento da ferramenta}

A ferramenta desenvolvida e apresentada neste artigo tem como objetivo apoiar a decisão para a superação de lacunas entre resultados de pesquisa e sua adoção, de forma alinhada com a abordagem micro de gaps. Tal ferramenta será doravante apresentada pela sigla TGRM, do inglês technological gap reduction methology. O termo lacuna é aqui definido como o conjunto de vazios e de barreiras que impedem ou dificultam que os resultados de projeto de $\mathrm{P} \& \mathrm{D}$ sejam apropriados, adotados e difundidos por meio da sua transformação em inovação.

É importante reforçar a ideia de que a aplicação da ferramenta TGRM não resolve, per se, o problema da falta de transferência, adoção e difusão de resultados tecnológicos, nem muito menos altera as taxas de introdução de novos produtos e processos. Ela equaciona, apoia e facilita a decisão de empresas e organizações que estejam dispostas a fazer isto.

Da mesma forma que na abordagem macro de gaps, a superação de lacunas deve ser precedida da necessidade, interesse e disposição em superá-las. Não há ferramenta ou método (plano micro), nem política ou estratégias (plano macro), que consiga elevar o nível de transferência, adoção e difusão de tecnologias sem que as partes envolvidas estejam dispostas a investir seus recursos nisto.

O desenvolvimento da ferramenta TGRM decorreu de demanda específica de uma empresa do setor elétrico brasileiro interessada em promover investimentos para reduzir gaps internos entre os resultados do seu esforço de P\&D e inovações de produto, processo e serviço.
A motivação da Empresa demandante estava relacionada à criação de modelos de gestão robustos para tornar os esforços de P\&D (ANEEL e além) mais efetivos, promovendo impactos positivos na empresa. O desenvolvimento da ferramenta TGRM ocorreu ao longo dos anos de 2012 e 2013, com base em uma estrutura de permanente validação, teste e revisão para garantir alinhamento com outras iniciativas de gestão da P\&D em andamento na Empresa.

As Figuras 1a e 1b ilustram o objetivo da ferramenta TGRM voltada à redução das lacunas existentes entre as tecnologias em estoque e sua apropriação por meio de produtos, processos e serviços. A ideia dessas Figuras é representar, de forma bastante simples, apesar de desafiadora, a intenção de reverter as inclinações das curvas de estoque e de apropriação do estoque de tecnologias - que têm se afastado ao longo do tempo - e tentar aproximá-las reduzindo assim a lacuna entre elas.

Essa redução deveria levar em conta vários critérios, dentre econômicos, produtivos, tecnológicos, gerencias, de mercado etc. Deveria, portanto, ser uma ferramenta capaz de contabilizar e comparar diferentes situações e oferecer ao tomador de decisão alternativas compatíveis com as ambições da organização.

Definido o objetivo da ferramenta, seu desenvolvimento foi organizado em quatro atividades. A primeira delas consistiu na definição das categorias de lacunas existentes no contexto dos projetos de $\mathrm{P} \& \mathrm{D}$ de empresas do setor elétrico nacional. Para tal foi realizada uma extensa revisão sobre a gestão e o marco regulatório da P\&D no setor elétrico nacional e suas implicações para a inovação, assim como sobre alguns conceitos básicos relacionados à gestão da $\mathrm{P} \& \mathrm{D}$ e transferência de tecnologia. Ademais, esta etapa envolveu uma análise dos resultados dos projetos de $\mathrm{P} \& \mathrm{D}$ da Empresa demandante nas últimas duas décadas (baseando-se em uma avaliação de resultados e impactos realizada em 2010 pela mesma equipe que desenvolveu e aplicou a presente ferramenta). Estes insumos deram as bases para a proposição das categorias de lacunas empregadas na ferramenta.

As frentes 2 e 3 foram realizadas de forma simultânea e interativa. A frente 2 correspondeu à elaboração de uma sequência integrada de questões
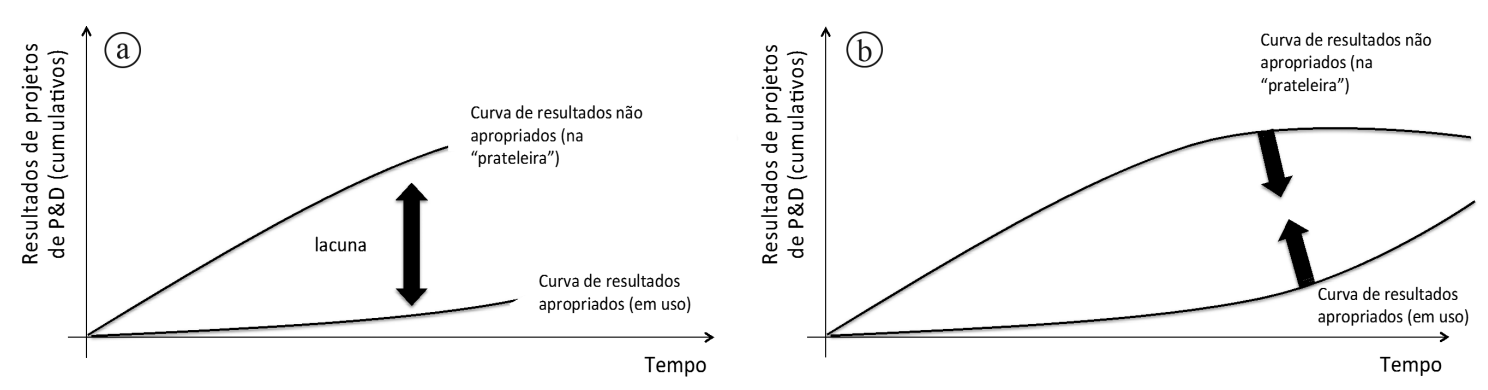

Figura 1. (a) Esquematização do problema. (b) Esquematização da solução. Fonte: Elaborado pelos autores. 
orientadas à caracterização de lacunas nas categorias propostas, com base na perspectiva de transferência, apropriação da tecnologia e geração de inovação.

A terceira frente, por sua vez, correspondeu à criação de um algoritmo relacionando as diferentes opções de respostas dadas à sequência de questões com distintos casos de apropriação, que por sua vez indicam opções nas formas de desenvolvimento, uso, licenciamento, produção e comercialização da tecnologia. Esta frente também definiu a descrição dos diversos casos que resultam da aplicação do algoritmo, assim como das recomendações de cada um deles para orientar a tomada de decisões no âmbito da Empresa.

A quarta e última frente correspondeu ao teste da ferramenta TGRM com três projetos de P\&D selecionados pela Empresa demandante para fins de validação. Com base nos resultados dos testes, foram realizados ajustes na ferramenta e a especificação para o desenvolvimento de um software, também testado e validado no âmbito da Empresa.

\section{TGRM: um método para apoiar a geração de inovações}

Esta seção apresenta uma visão integrada e detalhada da ferramenta TGRM proposta. Do ponto de vista prático, sugere-se que seu uso seja realizado por pessoas com conhecimento sobre os projetos de P\&D da empresa e sobre os aspectos tecnológicos e mercadológicos a ele relacionados, tais como gerentes de projeto, analistas de mercado e/ou pesquisadores (doravante denominados usuários). Sua aplicação pode ocorrer antes (ex-ante), durante ou depois (ex-post) do desenvolvimento dos projetos.

Ex-ante, por razões óbvias, já que a ferramenta ajuda a identificar e antecipar ações para atuar sobre o que efetivamente se deseja levar ao nível de inovação (produto, processo, serviço); durante, porque é possível que condições se alterem ao longo do projeto e exijam ajustes e redirecionamentos (fatos inesperados, reversão ou expansão de expectativas, resultados não esperados etc.); ex-post, para avaliar o estoque e também para revisar e, se necessário, ajustar ações previamente identificadas.

\subsection{Visão integrada da ferramenta TGRM}

A ferramenta desenvolvida pode ser dividida em quatro módulos, conforme esquematizado na Figura 2. Cada módulo é considerado uma operação, com uma "entrada" (input) e uma "saída" (output). Os módulos podem ser descritos como: (1) cadastro do projeto; (2) caracterização de lacunas; (3) algoritmo de análise; e (4) identificação do caso e indicação das ações pertinentes.

Em linhas gerais, no Módulo 1, o usuário cadastra informações sobre o projeto que será analisado para fins de armazenamento no sistema. Finalizado o cadastro, o usuário tem acesso ao Módulo 2, que consiste no roteiro para caracterização de lacunas científicas e tecnológicas, mercadológicas e gerencias do projeto. Depois da caracterização completa de lacunas, o usuário tem acesso ao Módulo 3, que permite a ele acionar o algoritmo de análise das lacunas. Depois de rodar o algoritmo, o usuário tem acesso ao Módulo 4, que consiste na "saída" (output) da ferramenta, contendo uma descrição do caso mais adequado em termos de perspectivas de apropriação, adoção e difusão de resultados do projeto em análise, assim como as ações pertinentes que devem ser tomadas para que o caso se viabilize.

Cada um dos módulos é detalhado nos itens a seguir.

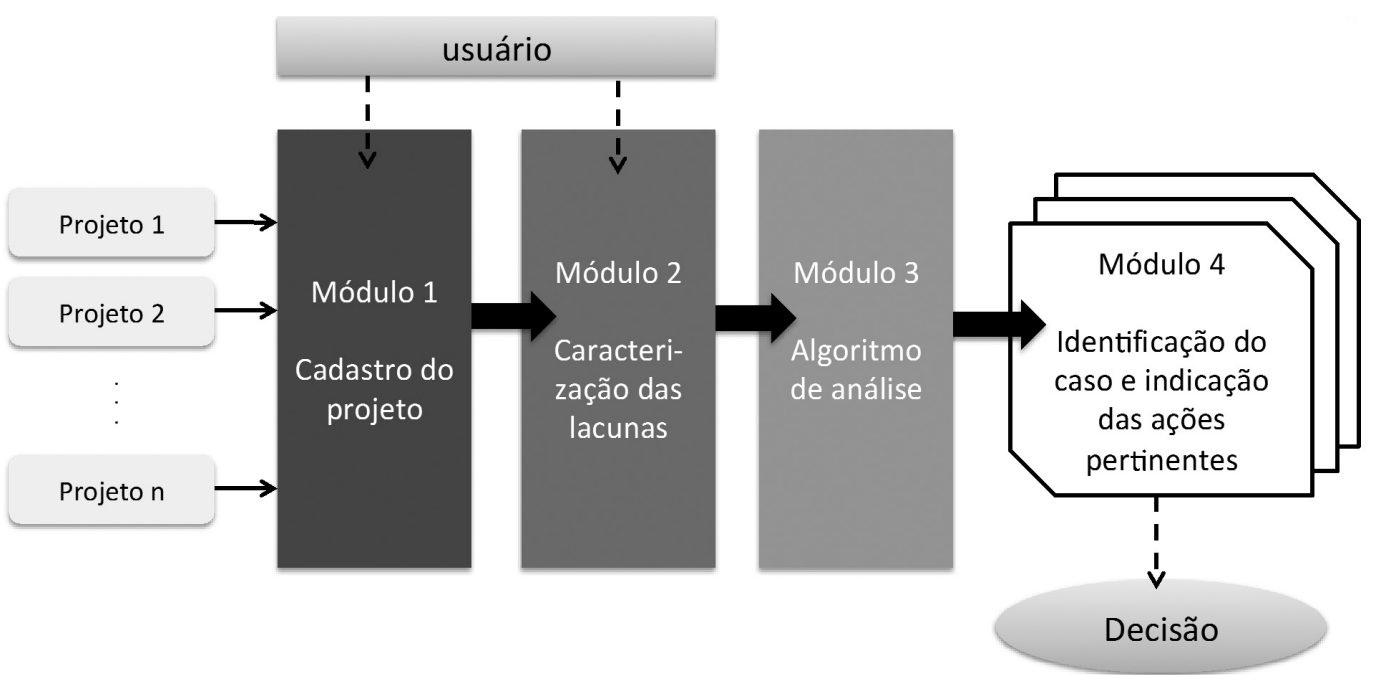

Figura 2. Esquema geral da ferramenta TGRM de apoio à decisão para apropriação dos resultados da P\&D. Fonte: Elaborado pelos autores. 


\subsection{Módulo 1: cadastro do projeto}

Este módulo consiste basicamente no cadastro de informações sobre o projeto de P\&D que será analisado para fins de armazenamento no sistema. Tais informações contemplam: identificação do projeto; período de execução; fase na cadeia de inovação de acordo com a classificação da ANEEL; parceiros de desenvolvimento (se pertinente); gerente do projeto; breve descrição do(s) principal(is) resultado(s) tecnológico(s) esperado(s) ou obtido(s).

Este cadastro é realizado por meio de uma interface com campos específicos nos quais o usuário deve inserir as informações.

\subsection{Módulo 2: caracterização das lacunas}

Foram consideradas no desenvolvimento da ferramenta TGRM três categorias de lacunas passíveis de ocorrência em projetos de $\mathrm{P} \& \mathrm{D}$ que visam à promoção da inovação, a saber:

i. Lacunas cientificas e tecnológicas são aquelas que se encontram entre o estágio de desenvolvimento de uma tecnologia e o estágio requerido para que a tecnologia possa ser levada a um produto ou a um processo e empregada internamente à empresa e/ou por ela comercializada. A redução deste tipo de lacunas envolve os aspectos técnicos e tecnológicos (às vezes científicos) que devem ser resolvidos para que uma tecnologia cumpra especificações normativas (se pertinente), esteja adaptada aos padrões de funcionamento dos sistemas de GTD e possua design adequado para utilização e/ou comercialização. ii. Lacunas mercadológicas são aquelas surgidas entre o estágio de desenvolvimento da tecnologia e a sua transformação em inovação de fato - compreendida como sua adoção, seja nos processos internos da empresa, seja por sua introdução no mercado, incluindo o emprego de ativos complementares (Teece, 1986) relacionados com sua produção, comercialização, distribuição e absorção.

iii. Lacunas gerenciais são aquelas que se estabelecem entre os esforços de gestão atuais e os esforços necessários para superar as lacunas científicas-tecnológicas e de mercado. Envolvem ações voltadas a garantir a disponibilidade de recursos financeiros, materiais e humanos necessários para o uso e exploração dos resultados dos projetos de $\mathrm{P} \& \mathrm{D}$ no âmbito organizacional.

O módulo de caracterização de lacunas consiste em uma sequência articulada de questões estruturadas a ser preenchida pelo usuário da ferramenta e que detalha os tipos de lacunas do projeto de $\mathrm{P} \& \mathrm{D}$ dentro das três categorias de análise. O Quadro 1 a seguir dá uma visão geral do conteúdo das questões.

As questões são organizadas de forma sequencial, com os condicionantes necessários (se [...] então [...]) e associadas a escalas nominais do tipo Likert ou a escalas optativas do tipo sim / não. Neste sentido, trata-se de um módulo de fácil preenchimento e salvamento.

\subsection{Módulo 3: algoritmo de análise}

O módulo 3 consiste no processamento realizado pela ferramenta para que, a partir de uma dada combinação de respostas no questionário, seja apontado

Quadro 1. Conteúdo geral das questões para a caracterização de lacunas.

\begin{tabular}{|c|c|c|}
\hline Lacunas científicas e tecnológicas & Lacunas mercadológicas & Lacunas gerenciais \\
\hline $\begin{array}{l}\text { - Estágio de maturidade } \\
\text { da tecnologia e tempo e } \\
\text { custo relacionado ao seu } \\
\text { desenvolvimento e/ou aquisição } \\
\text { - Nível de complexidade da } \\
\text { tecnologia } \\
\text { - Grau de inovatividade da } \\
\text { tecnologia } \\
\text { - Perspectivas de proteção da } \\
\text { propriedade intelectual } \\
\text { - Nível de dificuldade para cópia da } \\
\text { tecnologia } \\
\text { - Impactos do uso da tecnologia } \\
\text { - Existência de tecnologias } \\
\text { substitutas }\end{array}$ & $\begin{array}{l}\text { - Possibilidade de comercialização } \\
\text { da tecnologia e condições de } \\
\text { mercado } \\
\text { - Complexidade e investimento } \\
\text { necessário para produzir, } \\
\text { comercializar e distribuir bens/ } \\
\text { oferecer serviços baseados na } \\
\text { tecnologia } \\
\text { - Expectativa de retorno econômico } \\
\text { e de outros impactos a partir da } \\
\text { comercialização da tecnologia }\end{array}$ & $\begin{array}{l}\text { - Possibilidade de uso da tecnologia } \\
\text { em processos internos da empresa } \\
\text { - Disponibilidade de competências } \\
\text { internas para desenvolver, comprar, } \\
\text { absorver, produzir, comercializar e/ } \\
\text { ou distribuir a tecnologia } \\
\text { - Disponibilidade de parceiros, sua } \\
\text { expertise e o interesse da empresa } \\
\text { em desenvolvê-los } \\
\text { - Disponibilidade de recursos } \\
\text { financeiros (próprios ou não) para } \\
\text { desenvolver, comprar, absorver, } \\
\text { produzir, comercializar e/ou } \\
\text { distribuir a tecnologia }\end{array}$ \\
\hline
\end{tabular}

Fonte: Elaborado pelos autores. 
um caso mais adequado em termos de perspectivas de apropriação, adoção e difusão dos resultados do projeto de $\mathrm{P} \& \mathrm{D}$, assim como as ações pertinentes que devem ser tomadas para que a apropriação ocorra.

$\mathrm{O}$ algoritmo de análise que embasa o processamento foi construído como uma árvore decisória, em que as respostas encadeadas das questões levam a um determinado caso. Árvores decisórias são ferramentas de suporte à decisão, comumente empregadas em pesquisa operacional, que empregam o formato gráfico de árvore para modelar decisões (nós) e suas consequências, apoiando a identificação de estratégias (Loch \& e Bode-Greuel, 2001; Nowak \& Nowaka, 2013).

Estes casos são organizados com base em três eixos principais: (i) desenvolvimento autônomo da tecnologia ( $\mathrm{P} \& \mathrm{D}$ interno) ou desenvolvimento externo (aquisição de P\&D) ou aquisição (compra) - a chamada decisão make or buy; (ii) uso (ou não) da tecnologia em processos internos da empresa; (iii) licenciamento e/ou produção e/ou comercialização da tecnologia. Conforme destacado anteriormente, tais decisões são a base para o problema que se coloca atualmente na gestão da $\mathrm{P} \& \mathrm{D}$ no setor elétrico brasileiro.

Vale ressaltar aqui que a ideia do uso da tecnologia em processos internos da empresa diz respeito à sua absorção nas mais variadas atividades meio e finalística das concessionárias como forma de melhorar a eficiência e trazer ganhos em termos de produtividade, qualidade e custo. $\mathrm{O}$ licenciamento, por sua vez, envolve o uso ou exploração comercial por terceiros da tecnologia desenvolvida e protegida por algum tipo de instrumento de propriedade intelectual, com a perspectiva de recebimento de royalties.

Por fim, a ideia de produção e comercialização, quando pensada para o caso do setor elétrico, está intrinsicamente ligada a novos modelos de negócio que vão além das funções GTD (p.e. produção e comercialização de equipamentos elétricos ou serviços de análise de dados de consumo em um determinado segmento ou região), podendo ser explorada pela concessionária ou por terceiros. A combinação desses eixos em suas diferentes situações levou à definição de 22 casos possíveis e ilustrados na Figura 3, nos quais um resultado de pesquisa pode ser categorizado.

As primeiras 10 combinações correspondem a casos nos quais o desenvolvimento da tecnologia é realizado de forma autônoma pela empresa, combinando diferentes possibilidades para uso, licenciamento, produção e comercialização da tecnologia. As combinações de 11 a 20 correspondem a situações similares aos primeiros 10 casos em termos de uso, licenciamento, produção e comercialização da tecnologia, mas que ocorrem com a aquisição de P\&D. Finalmente, os casos 21 e 22 estão relacionados com alternativas de compra da tecnologia, combinadas com opções de uso corporativo ou produção e comercialização para o mercado.

\subsection{Módulo 4: identificação do caso e indicação das ações pertinentes}

O Módulo 4 consiste na "saída" (output) da ferramenta, contendo a descrição do caso indicado a partir do processamento do módulo anterior, assim como as ações pertinentes que devem ser tomadas para que a apropriação, adoção ou difusão ocorra.

\subsection{Aplicando a ferramenta TGRM}

A ferramenta foi validada rodando-se os resultados de três projetos de P\&D já finalizados da Empresa demandante. A seleção dos projetos foi feita pela
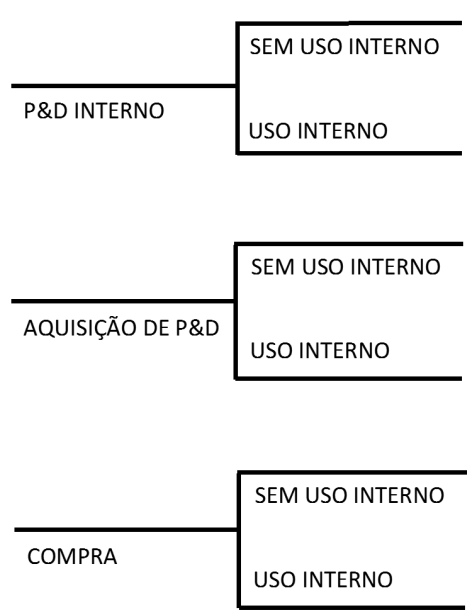

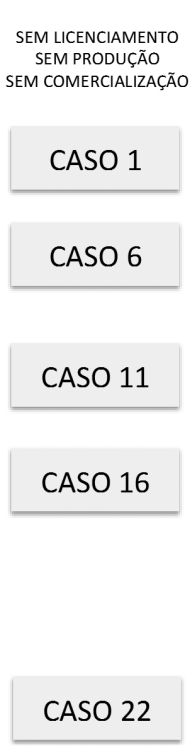

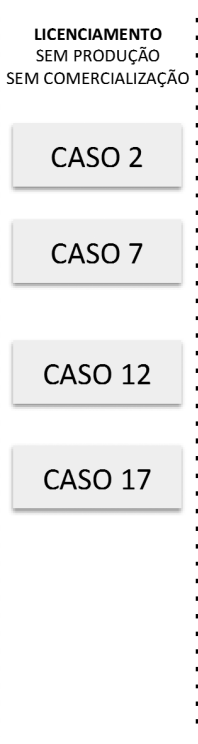
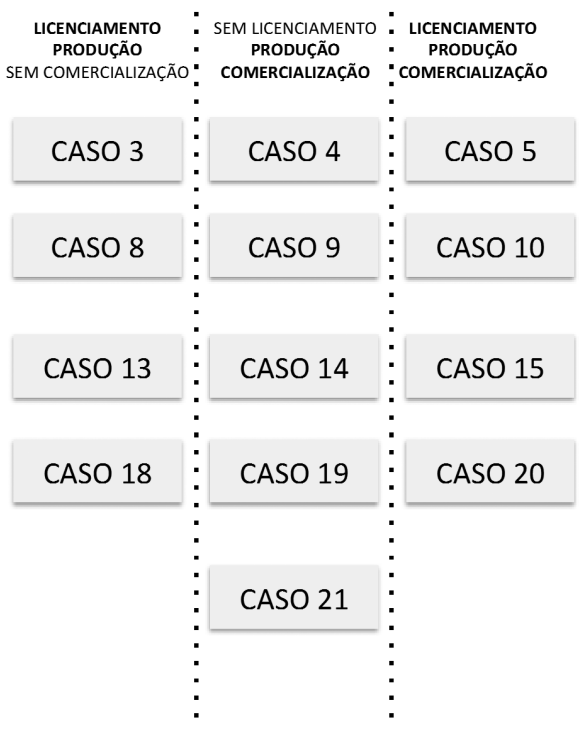

Figura 3. Casos de apropriação dos resultados de projetos de P\&D. Fonte: Elaborado pelos autores. 
própria Empresa com base nas expectativas de inovação relacionadas aos projetos. Apresentam-se a seguir dois exemplos de aplicação (doravante denominados de projeto $\alpha$ e $\beta$ ) para melhor ilustrar tanto o funcionamento quanto os resultados alcançados pelo método.

O projeto $\alpha$ foi executado entre 2006 e 2009 pela Empresa em parceria com uma universidade e uma empresa da área de engenharia e teve como objetivo desenvolver protótipos de um equipamento para aplicação industrial. Os protótipos foram testados e certificados e houve aproximação com uma empresa não envolvida no desenvolvimento, mas com interesse na produção e comercialização do equipamento.

As respostas obtidas no Módulo de caracterização de lacunas indicaram a ausência de perspectiva para uso da tecnologia desenvolvida em processos internos da Empresa (pois trata-se de um equipamento para aplicação industrial), assim como a falta de recursos e competências da própria Empresa em produzir e comercializar o equipamento no mercado. No entanto, a existência de um importante potencial de mercado no Brasil sugeriu o licenciamento da tecnologia como uma boa alternativa, o que permitiria a geração de receitas advindas de royalties. O processamento das respostas do módulo apontou para o caso 12 . A Figura 4 simula a "saída" da ferramenta após o

\begin{tabular}{|c|c|}
\hline Descrição - Caso 12 & Ações - Caso 12 \\
\hline $\begin{array}{l}\text { Neste caso, temos uma tecnologia difícil de } \\
\text { ser incorporada na Empresa. A tecnologia é } \\
\text { complexa para produzir/comercializar/ } \\
\text { distribuir, é suscetivel de transferência/venda, } \\
\text { com perspectivas de proteção à propriedade } \\
\text { intelectual e grande potencial de mercado e } \\
\text { de retorno econômico a partir da } \\
\text { comercialização indireta (por terceiros). o } \\
\text { custo de desenvolvimento no Brasil é menor } \\
\text { que no mundo ou há recursos financeiros } \\
\text { internos/externos para o desenvolvimento. Há } \\
\text { interesse em estabelecer parcerias para o } \\
\text { desenvolvimento, mas não para produzir/ } \\
\text { comercializar/distribuir a tecnologia. Não há } \\
\text { competências internas para desenvolver/ } \\
\text { produzir/comercializar/distribuir a tecnologia. }\end{array}$ & $\begin{array}{l}\text { - Garantir a proteção da propriedade } \\
\text { intelectual. } \\
\text { Caso o projeto não tenha sido iniciado, } \\
\text { buscar desde seu início parceiros } \\
\text { interessados no desenvolvimento } \\
\text { conjunto e no licenciamento da } \\
\text { tecnologia. Acordar com eles desde logo } \\
\text { as condições de proteção da propriedade } \\
\text { intelectual dos resultados que serão } \\
\text { gerados. } \\
\text { Caso o projeto esteja finalizado, encontrar } \\
\text { empresas interessadas em ter a } \\
\text { tecnologia licenciada para produção e } \\
\text { comercialização. }\end{array}$ \\
\hline
\end{tabular}

Figura 4. Caso de apropriação/adoção/difusão para o projeto $\alpha$. Fonte: Elaborado pelos autores.

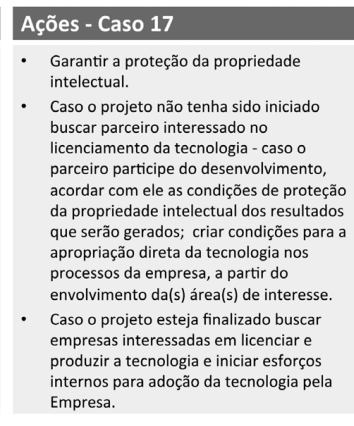

Figura 5. Caso de apropriação/adoção/difusão para o projeto $\beta$. Fonte: Elaborado pelos autores. preenchimento do questionário e processamento das respostas para o projeto $\alpha$.

O projeto $\boldsymbol{\beta}$ foi executado entre 2004 e 2006 pela Empresa em parceria com uma universidade e teve como objetivo produzir protótipos de equipamentos a serem utilizados na instalação e manutenção de serviços de energia.

As respostas obtidas no Módulo 2 indicaram uma perspectiva para uso das tecnologias desenvolvidas em processos internos da empresa, apesar da falta de interesse, recursos e competências para produção e comercialização no mercado. No entanto, a existência de um importante potencial de mercado no Brasil por parte de outras empresas de energia sugere, assim como no caso anterior, o licenciamento da tecnologia como uma boa alternativa para a Empresa, o que permitirá a ela ser usuária do equipamento e, ao mesmo tempo, obter receitas advindas de royalties do licenciamento. O processamento das respostas do módulo apontou para o caso 17. A Figura 5 simula a "saída" da ferramenta para o projeto $\boldsymbol{\beta}$.

\section{Discussão e conclusões}

Conforme discutido na caracterização do setor elétrico nacional, várias tecnologias têm sido desenvolvidas nestes anos de investimentos em P\&D por parte das empresas do setor elétrico nacional e de seus parceiros no âmbito do Programa de P\&D da ANEEL (Fernandino \& Oliveira, 2010; Pompermayer et al., 2011; Boer, 2013).

Todavia, a incorporação destas mesmas tecnologias aos processos produtivos das empresas e/ou sua comercialização (por parte das empresas ou por terceiros) tem se mostrado bastante frágil, justamente pela falta de incentivos para que este fenômeno ocorra. Assim, apesar do investimento, os benefícios para as empresas, para o setor e para a sociedade têm sido restritos (Pompermayer et al., 2011). Vale destacar que esta constatação não significa que há insucesso na execução dos projetos de P\&D das empresas: há controle sobre os resultados gerados (particularmente em razão do risco regulatório), sejam estes resultados novos conceitos, metodologias, componentes, equipamentos, sistemas, entre outros. A limitação tem sido justamente em relação à apropriação social e econômica destes resultados.

Se por um lado esta situação revela uma fragilidade dos modelos gerenciais que vêm sendo adotados por estas empresas devido, em parte, à carência de ferramentas que apoiem as relações estabelecidas entre elas e seus parceiros de P\&D e, mais do que isso, à tomada e implementação de decisões acerca da apropriação dos resultados gerados, por outro lado há razões de fundo para o relativo insucesso do Programa de P\&D ANEEL na geração de benefícios econômicos e sociais. Tais razões estão associadas à própria falta de estímulo das empresas para inovar, 
uma vez que historicamente elas não têm vislumbrado os benefícios que podem obter a partir destes esforços (Salles-Filho, 2010; Amaral, 2012; Boer et al., 2014).

A discussão sobre a efetividade da política de P\&D no setor tem sido bastante ampla nos últimos anos e são vários os argumentos empregados para defender o modelo atual ou propor mudanças. Embora não seja o objetivo deste trabalho aprofundar tal debate, cabe destacar que, sob a ótica da gestão da P\&D, é bastante pertinente levar em consideração o quão eficiente este modelo descentralizado tem sido, tanto para a identificação de lacunas tecnológicas existentes e definição de temas prioritários para orientar a P\&D, quanto para a execução propriamente dita da P\&D e apropriação de seus resultados. Por um lado, é possível dizer que a política é alinhada com boas práticas de gestão da inovação e de inovação aberta (Tidd et al., 2005; Chesbrough, 2006), uma vez que se fundamenta na importância de estabelecer e reforçar estruturas próprias de gestão tecnológica nas empresas, estimulando parcerias para P\&D e inovação, principalmente com fabricantes de materiais e equipamentos (ANEEL, 2012). Por outro lado, é possível dizer que a política não garante boas condições de apropriabilidade para os investimentos em P\&D e não considera a própria dinâmica supplier dominated do setor, criando redundâncias e desperdícios, que poderiam ser minimizados por uma gestão centralizada dos recursos (Pompermayer et al., 2011; Amaral 2012; Boer et al., 2014).

Independentemente disso, é fato que as transformações recentes do marco regulatório do setor a partir de 2008 têm modificado um pouco a percepção dos estímulos para inovar por parte das empresas. De forma complementar, os movimentos de estímulo à inovação no País, por meio de políticas e diversificação das fontes de financiamento, têm também modificado esta percepção. O resultado mais imediato disso tem sido justamente um reposicionamento das áreas de P\&D das empresas do setor elétrico (que têm passado para níveis estratégicos superiores e têm ampliado seu escopo para além do investimento compulsório de P\&D ANEEL), assim como a sofisticação de seus modelos de gestão, como demonstrado por Boer et al. (2014) em seu trabalho de avaliação das estruturas de gestão de P\&D de empresas GTD.

Esta sofisticação tem caminhado em várias direções, com destaque para instrumentos tradicionais de gestão da inovação, tais como prospecção de tendências futuras, priorização de temas e projetos, avaliação ex-ante e ex-post de projetos e tecnologias, gestão da propriedade intelectual e de colaborações (na perspectiva da inovação aberta).

Nota-se, no entanto, uma carência de instrumentos para se lidar com os estoques de resultados da P\&D das empresas, aqui compreendidos como as tecnologias por elas desenvolvidas e que permanecem na "prateleira", assim como para garantir a apropriação futura dos resultados gerados nos projetos, seja esta apropriação baseada no modelo de negócio atual das empresas, seja ela baseada também em novos modelos de negócio, voltados ao licenciamento de tecnologias (para o setor elétrico e outros setores) ou ainda à produção e comercialização de novos bens e serviços.

A ferramenta TGRM proposta neste trabalho visa atuar justamente neste espaço negligenciado no âmbito da gestão da inovação nas empresas do setor de energia elétrica, no intuito de gerar diretrizes para lidar com o estoque atual e potencial de tecnologias no contexto de um P\&D mais ampliado e estratégico, caracterizando lacunas de diferentes naturezas que impedem ou dificultam a necessária ponte entre P\&D e mercado e indicando soluções para superá-las.

A vantagem da ferramenta é apresentar, por meio de um procedimento simples, orientações claras para a desejada apropriação de resultados por meio de combinações entre a perspectiva mais adequada para o seu desenvolvimento ou compra (make or buy), perspectiva de uso interno da tecnologia pelas empresas e perspectiva de comercialização.

Há de se considerar, todavia, uma limitação importante do presente estudo. Apesar de testada e validada com projetos de $\mathrm{P} \& \mathrm{D}$ reais, não há constatação empírica de como a metodologia TGRM efetivamente contribui para a redução e superação de lacunas entre a P\&D e a inovação no setor elétrico nacional. Esta é uma resposta que apenas poderá ser dada no médio prazo, a partir de decisões internas da empresa relacionadas à incorporação da ferramenta no cotidiano da gestão e, mais do que isso, das decisões estratégicas que decorrem de seu uso.

Por fim, muito embora a metodologia e a ferramenta aqui apresentadas tenham se pautado nos desafios do setor elétrico brasileiro, cabe enfatizar que elas têm potencial de aplicabilidade para outros setores econômicos, desde que tais setores compartilhem com o setor elétrico características peculiares em termos das dificuldades de superação de lacunas entre os resultados de projetos e P\&D e os impactos efetivos da inovação, assim como para outros tipos de organizações (que não as privadas com fins lucrativos), que têm como missão a realização de P\&D e que também enfrentam desafios relacionados à geração de benefícios a partir destes resultados, especialmente universidades e institutos públicos de pesquisa.

\section{Referências}

Abramovitz, M. (1986). Catching up, forging ahead, and falling behind. The Journal of Economic History, 46(2), 385-406. http://dx.doi.org/10.1017/S0022050700046209. 
Amaral, G. S. G. (2012). A pesquisa e desenvolvimento no setor elétrico brasileiro: uma investigação da politica tecnológica para o setor com base na teoria evolucionária da mudança técnica (Dissertação de mestrado). Escola Politécnica da Universidade de São Paulo, São Paulo.

Amesse, F., \& Cohendet, P. (2001). Technology transfer revisited from the perspective of the knowledge-based economy. Research Policy, 30(9), 1459-1478. http:// dx.doi.org/10.1016/S0048-7333(01)00162-7.

Agência Nacional de Energia Elétrica - ANEEL. (2008). Manual do Programa de Pesquisa e Desenvolvimento Tecnológico do Setor de Energia Elétrica. Brasília: ANEEL.

Agência Nacional de Energia Elétrica - ANEEL. (2009). Atlas de energia elétrica do Brasil. Brasília: ANEEL.

Agência Nacional de Energia Elétrica - ANEEL. (2012). Manual do Programa de Pesquisa e Desenvolvimento Tecnológico do Setor de Energia Elétrica. Brasília: ANEEL.

Bell, M., \& Pavitt, K. (1993). Technological accumulation and industrial growth: contrasts between developed and developing countries. Industrial and Corporate Change, 2(2), 157-210. http://dx.doi.org/10.1093/icc/2.1.157.

Boer, D. C. (2013). Gestão de pesquisa, desenvolvimento e inovação no setor elétrico brasileiro (Tese de doutorado). Universidade Estadual de Campinas, Campinas.

Boer, D. C., Salles-Filho, S. L. M., \& Bin, A. (2014). R\&D and innovation management in the brazilian electricity sector: the regulatory constraint. Journal of Technology Management \& Innovation, 9(1), 44-56. http://dx.doi. org/10.4067/S0718-27242014000100004.

Brasil. (2000, 24 de julho). Lei $n^{\circ}$ 9.991, de 24 de julho de 2000. Dispõe sobre realização de investimentos em pesquisa e desenvolvimento e em eficiência energética por parte das empresas concessionárias, permissionárias e autorizadas do setor de energia elétrica, e dá outras providências. Brasília, DF: Diário Oficial da República Federativa do Brasil.

Campos, I. A. (2007). Potencialidades e energias renováveis no brasil: perspectiva eólica. In I Seminário do CentroOeste de Energias Renováveis. Goiânia.

Castellacci, F. (2002). Technology-gap and cumulative growth: models, results and performances. In DRUID Winter Conference. Aalborg.

Castellacci, F. (2008). Technological paradigms, regimes and trajectories: manufacturing and service industries in a new taxonomy of sectoral patterns of innovation. Research Policy, 37(6-7), 978-994. http://dx.doi. org/10.1016/j.respol.2008.03.011.

Castellacci, F. (2009). The interactions between national systems and sectoral patterns of innovation: a crosscountry analysis of Pavitt's taxonomy. Journal of Evolutionary Economics, 19(3), 321-347. http://dx.doi. org/10.1007/s00191-008-0113-9.
Chesbrough, H. (2006). Open innovation: the new imperative for creating and profiting from technology. Harvard: Harvard Business School Press.

Cohen, W. M., \& Levinthal, D. A. (1990). Absorptive capacity: a new perspective on learning and innovation. Administrative Science Quarterly, 35(1), 128-152. http:// dx.doi.org/10.2307/2393553.

Cooper, R. G. (2000). Doing it right: winning with new projects. Ivey Business Journal, 4, 54-60.

Cunha, J. C., Silva, E., Dias, C., \& Girardi, S. (2008). Sistema setorial de inovação de energia elétrica no brasil: estrutura e trajetórias. In Anais do XXV Simpósio de Gestão da Inovação Tecnológica. (pp. 1-16). Brasília: ANPAD.

Defeuilley, C., \& Furtado, A. T. (2000). Impacts de l'ouverture à la concurrence sur la R\&D dans le secteur électrique. Annals of Public and Cooperative Economics, 71(1), 5-28. http://dx.doi.org/10.1111/1467-8292.00131.

Dodgson, M., Gann, D., \& Salter, A. (2008). The management of technological innovation: strategy and practice. New York: Oxford University Press.

Fernandino, J. A., \& Oliveira, J. L. (2010). Arquiteturas organizacionais para a área de P\&D em empresas do setor elétrico brasileiro. Rev. adm. contemp, 14(6), 1073-1093. http://dx.doi.org/10.1590/S1415-65552010000700006.

Gomes, C. M., Kruglianskas, I., \& Scherer, F. L. (2011). Gestão das fontes externas de informação: uma análise dos fatores que influenciam o desempenho inovador. Gestão \& Produção, 18(4), 897-910. http://dx.doi. org/10.1590/S0104-530X2011000400015.

Herrera, A. (1971). Ciencia y politica en América Latina. México: Siglo XXI.

Herrera, A. (1982). A grande jornada. São Paulo: Paz e Terra.

Jamasb, T., \& Pollitt, M. (2011). Electricity sector liberalisation and innovation: an analysis of the UK's patenting activities. Research Policy, 40(2), 309-324. http://dx.doi.org/10.1016/j.respol.2010.10.010.

Lavie, D., Haunschild, P. R., \& Khanna, P. (2012). Organizational differences, relational mechanisms, and alliance performance. Strategic Management Journal, 33(13), 1453-1479. http://dx.doi.org/10.1002/smj.1987.

Lichtenthaler, U. (2011). The evolution of technology licensing management: identifying five strategic approaches. $R \& D$ Management, 41(2), 173-189. http://dx.doi.org/10.1111/j.1467-9310.2011.00635.x.

Loch, C. H., \& Bode-Greuel, K. (2001). Evaluating growth options as sources of value for pharmaceutical research projects. $R \&$ D Management, 31(2), 231-248. http:// dx.doi.org/10.1111/1467-9310.00212.

Malerba, F., \& Orsenigo, L. (1997). Technological regimes and sectoral patterns of innovation activities. Industrial and Corporate Change, 6(1), 83-117. http://dx.doi. org/10.1093/icc/6.1.83. 
Mansfield, E. (1984). R\&D and innovation: some empirical findings. In $\mathrm{Z}$. Griliches (Ed.). R\&D, patents and productivity (pp. 127-154). Chicago: University of Chicago Press.

Margolis, R. M., \& Kammen, D. M. (1999). Underinvestment: the energy technology and R\&D policy challenge. Science, 285(5428), 690-692. http://dx.doi.org/10.1126/ science.285.5428.690. PMid:10426983.

Miozzo, M., \& Soete, L. (2001). Internationalization of Services: a technological perspective. Technological Forecasting and Social Change, 67(2), 159-185. http:// dx.doi.org/10.1016/S0040-1625(00)00091-3.

Nowak, M., \& Nowaka, B. (2013). An application of the multiple criteria decision tree in project planning. Procedia Technology, 9, 826-835. http://dx.doi.org/10.1016/j. protcy.2013.12.092.

Pavitt, K. (1984). Sectoral patterns of technological change: towards a taxonomy and a theory. Research Policy, 13(6), 343-373. http://dx.doi.org/10.1016/00487333(84)90018-0.

Perez, C. (1985). Microelectronics, long waves and technical change: new perspectives for developing countries. World Development, 13(3), 441-463. http://dx.doi. org/10.1016/0305-750X(85)90140-8.

Perez, C., \& Soete, L. (1988). Catching up in technology: entry barries and windows of opportunity. In G. Dosi, C. Freeman, R. Nelson, G. Silverberg \& L. L. Soete (Eds.), Technical change and economic theory. London: Francis Pinter. pp. 458-479.

Phaal, R., Farrukh, C. J. P., \& Probert, D. R. (2006). Technology management tools: concept, development and application. Technovation, 26(3), 336-344. http:// dx.doi.org/10.1016/j.technovation.2005.02.001.

Pompermayer, F. M., De Negri, F., \& Cavalcante, L. R. (2011). Inovação tecnológica no setor elétrico brasileiro: uma avaliação do programa $P \& D$ regulado pela Aneel. Brasília: Instituto de Pesquisa Econômica Aplicada.
Rice, M. P., Leifer, R., \& O’Connor, G. C. (2002). Commercializing discontinuous innovations: bridging the gap from discontinuous innovation project to operations. IEEE Transactions on Engineering Management, 49(4), 330-340. http://dx.doi.org/10.1109/TEM.2002.806721.

Salles-Filho, S. L. M. (2010, 22 de fevereiro). Tecnologia e inovação no setor elétrico. Folha de São Paulo, p. 3.

Teece, D. (1986). Profiting from technological innovation: implications for integration, collaboration, licensing and public policy. Research Policy, 15(6), 285-305. http:// dx.doi.org/10.1016/0048-7333(86)90027-2 .

Teece, D. (2010). A tribute to Oliver Williamson: Williamson's impact on the theory and practice of management. California Management Review, 52(2), 167-176. http:// dx.doi.org/10.1525/cmr.2010.52.2.167.

Tenório, J. B. (2009). Indicadores e métricas: ferramentas para avaliação de resultados de $P \& D$ visando a inovação no setor elétrico (Dissertação de mestrado). Pontifícia Universidade Católica do Rio de Janeiro.

Tidd, J., Bessant, J., \& Pavitt, K. (2005). Managing innovation: integrating technological, market and organizational change (3rd. ed.).Chichester: John Wiley \& Sons.

Vianna, L. F. L. (2004). As associações setoriais e um passeio pela história da energia elétrica no Brasil. In Grupo CanalEnergia. Setor elétrico brasileiro - passado e futuro: 10 anos. Rio de Janeiro: CanalEnergia.

Wigren-Kristoferson, C., Gabrielsson, J., \& Kitagawa, F. (2011). Mind the gap and bridge the gap: research excellence and diffusion of academic knowledge in Sweden. Science \& Public Policy, 38(6), 481-492. http://dx.doi.org/10.3152/030234211X12960315267859.

Youtie, J., Hicks, D., Shapira, P., \& Horsley, T. (2012). Pathways from discovery to commercialisation: using web sources to track small and medium-sized enterprise strategies in emerging nanotechnologies. Technology Analysis and Strategic Management, 24(10), 981-995. http://dx.doi.org/10.1080/09537325.2012.724163. 